\title{
Más allá del reconocimiento: la evaluación externa de programas educativos
}

\begin{abstract}
A lo largo de 22 años, la Universidad Autónoma de Aguascalientes ha promovido que sus programas educativos participen en procesos de evaluación externa, tanto nacionales como internacionales, con la finalidad de asegurar su calidad. Estos procesos son ejecutados por organismos de amplio prestigio que conjuntan a pares académicos de diversas instituciones del país o del extranjero, quienes con una visión objetiva, analizan la situación de los programas y aportan sugerencias de mejora. Como resultado de estos procesos, la Universidad ha obtenido un sinnúmero de reconocimientos que la han colocado como un referente de calidad a nivel nacional.
\end{abstract}

Con el paso del tiempo, además de los reconocimientos obtenidos y de las recomendaciones que los organismos externos han aportado para la mejora continua, esta participación ha dejado importantes aprendizajes para toda la comunidad universitaria. En esta ocasión se ha conjuntado la opinión de siete profesores universitarios que han participado directamente en los procesos de evaluación externa, a quienes se entrevistó personalmente, solicitando que compartieran sus experiencias y los aprendizajes adquiridos.

Con base en sus aportaciones es posible definir siete categorías de beneficios que, en opinión de los entrevistados, se han generado a partir de los procesos de evaluación externa, tanto la que se ha realizado con organismos nacionales como internacionales; estas categorías pueden identificarse de la siguiente manera: responsabilidad, conocimiento de la Universidad Autónoma de Aguascalientes, accción y transformación, trabajo colaborativo, crecimiento profesional y personal, mejora continua, e identidad y sentido del porqué hacer las cosas. A continuación se desarrollan algunas de las ideas más relevantes en relación con estas categorías de beneficios identificadas.

\section{Responsabilidad}

Participar en los procesos de evaluación externa implica un gran compromiso más allá de adquirir una obligación con la institución, ya que es donde se plasma la viva imagen de cada una de las carreras, sus bondades y sus áreas de mejora. Parafraseando a la maestra Irma Adriana Castro Gallo, Jefa del Departamento de Química:

[...] al inicio uno puede dudar de los procesos de evaluación externa, pero cuando nos damos cuenta de su transcendencia e importancia, asumimos el rol que nos corresponde y tomamos nuestra responsabilidad, no sólo con el proceso de evaluación en sí, sino que tomamos en nuestras manos el proyecto de mejora anteponiendo siempre nuestro compromiso como miembros de la comunidad universitaria.

1 Entrevista a Irma Adriana Castro Gallo, realizada por Cardona, E. y Fresán, D., UAA, 06 de enero de 2016. 


\section{Conocimiento de la Universidad Autónoma de Aguascalientes y sus funciones}

Todos los entrevistados coincidieron en resaltar que participar en los procesos de evaluación externa les ha permitido conocer mejor a la Universidad, y comprender cuál es la función de cada una de las direcciones y departamentos que los apoyaron durante el trayecto. Retomando los comentarios de la licenciada Tania Melina Cervantes Rincón, Coordinadora de la Unidad de Atención e Investigación en Psicología, se transcribe: "[antes pensaba] que cada quien hacía su trabajo desde su trinchera", pero a través de los procesos de evaluación externa es posible "conocer la ardua labor que realizan todas las áreas y la vasta información que se genera. Participar en el proceso me ha permitido aprender de la organización". ${ }^{2}$

\section{Acción y transformación}

Las evaluaciones externas permiten comprender, desde una visión objetiva e imparcial, las fortalezas y áreas de mejora de programas educativos, tal como comentó el doctor Jorge Luis López Rodríguez, Jefe del Departamento de Cirugía: "al coordinar estos procesos de evaluación se adquiere una mejor visión y conocimiento del programa educativo y de los fines de la formación profesional; sin duda los estudiantes resultan beneficiados por los cambios positivos que se promueven a partir de la evaluación". ${ }^{3}$

Todos los entrevistados detallaron diversas actividades o acciones que han emprendido a partir de la evaluación, y principalmente a raíz de las recomendaciones que los organismos evaluadores han aportado; muchas de ellas contribuyen directamente a fortalecer el programa educativo o a subsanar sus áreas de oportunidad, algunas de las más recurrentes son:

2 Entrevista a Tania Melina Cervantes Rincón, realizada por Cardona, E. y Fresán, D., UAA, 07 de enero de 2016.

3 Entrevista a Jorge Luis López Rodríguez, realizada por Cardona, E. y Fresán, D., UAA, 07 de enero de 2016. a. Se han incluido las recomendaciones de evaluadores especialistas en la revisión y actualización de los planes de estudio.

b. Se ha incrementado la habilitación de los profesores en programas de posgrado y su participación en cursos y eventos de formación y actualización pedagógica y disciplinar para promover las mejores prácticas docentes.

c. Se han mejorado los instrumentos y procedimientos de evaluación del aprendizaje.

d. Se han gestionado recursos para mejorar la infraestructura y los servicios.

e. Se han establecido las bases para avanzar en la investigación, entre otras.

\section{Trabajo colaborativo}

Los entrevistados coinciden al resaltar que se ha fortalecido la capacidad de trabajar en equipo al interior de los comités de autoevaluación, y en sinergia con todas las áreas académicas y de la institución que de alguna manera apoyan los procesos, incluso "se mejora el ambiente laboral". ${ }_{4}^{4}$ Tal como lo comentó el maestro Ricardo Mosqueda Villalobos, Jefe del Departamento de Optometría y el maestro José Trinidad Marín Aguilar, Coordinador de Evaluación y Acreditación del Centro de Ciencias Económicas y Administrativas, quien expresó que: "se genera un ambiente de igualdad, todos trabajamos al mismo nivel, no hay jerarquías cuando se pretende alcanzar las metas propuestas". 5

\section{Crecimiento profesional y personal}

Una percepción generalizada de los profesores entrevistados es que a través de los procesos de evaluación externa han experimentado un crecimiento profesional y personal que puede notarse a través del desarrollo de mayores capacidades para: or-

4 Entrevista a Ricardo Mosqueda Villalobos, realizada por Cardona, E. y Fresán, D., UAA, 06 de enero de 2016.

5 Entrevista a José Trinidad Marín Aguilar, realizada por Cardona, E. y Fresán, D., UAA, 07 de enero de 2016. 
uno quiera más a la institución"; 9 el doctor Jorge Luis López expresó: "He aprendido a hacer mis actividades con entrega, pasión e interés"; ${ }^{10}$ el maestro Francisco López comentó: "Los profesores han adquirido una mayor identidad con la Universidad, lo que les ha permitido participar con una mejor actitud en los procesos de evaluación"; ${ }^{11}$ el maestro Ricardo Mosqueda dijo: "Me he dado cuenta de la gran importancia de mi papel en el apoyo a los estudiantes y en la coordinación del Departamento"; 12 el maestro José Trinidad Marín concluyó que: "La evaluación externa es la fuente de un mayor prestigio institucional y es un orgullo pertenecer a esta institución"; ${ }^{13}$ finalmente, la maestra Carolina Arroyo agregó: "Este tipo de procesos son muy buenos para fortalecer la identidad [de los alumnos y profesores] con su carrera y con la Universidad". ${ }^{14}$

\section{Conclusiones}

La evaluación externa de la calidad de los programas educativos es un ejercicio que va más allá de la búsqueda de un reconocimiento público a la calidad, pues es un insumo de gran valor para propiciar los grandes cambios que se requieren al interior de las instituciones educativas.

La experiencia obtenida con el paso de los años en la participación de los procesos de evaluación externa abre una puerta para que toda la comunidad universitaria se involucre en ellos con una actitud abierta al cambio, con responsabilidad ética y social, privilegiando la formación humanista que rige a la institución, lo cual implica entrar en una espiral de aprendizajes y conocimientos tanto personales como institucionales, que finalmente cul-

9 Entrevista a Irma Adriana Castro Gallo, realizada por Cardona, E. y Fresán, D., UAA, 06 de enero de 2016.

10 Entrevista a Jorge Luis López Rodríguez, realizada por Cardona, E. y Fresán, D., UAA, 07 de enero de 2016.

11 Entrevista a Francisco Javier López López, realizada por Cardona, E. y Fresán, D., UAA, 07 de enero de 2016.

12 Entrevista a Ricardo Mosqueda Villalobos, realizada por Cardona, E. y Fresán, D., UAA, 06 de enero de 2016.

13 Entrevista a José Trinidad Marín Aguilar, realizada por Cardona, E. y Fresán, D., UAA, 07 de enero de 2016.

14 Entrevista a Carolina Arroyo Solórzano, realizada por Cardona, E. y Fresán, D., UAA, 06 de enero de 2016. minan en la aplicación de procesos de innovación y mejora continua, aspectos que son fundamentales para los retos que enfrenta la educación actual y que permiten revalorar el significado y trascendencia de la evaluación externa.

Agradecemos a los profesores participantes, su tiempo y sus aportaciones para la integración de este artículo, así como a todos aquellos que directa e indirectamente han contribuido a la evaluación externa.

\section{Fuentes testimoniales}

Arroyo, C. (2016). Entrevista personal "Aprendizajes de participar en los procesos de evaluación externa" con la licenciada Carolina Arroyo Solórzano. [Grabación de audio]. UAA, 06 de enero de 2016.

Castro, I. (2016). Entrevista personal "Aprendizajes de participar en los procesos de evaluación externa" con la maestra Irma Adriana Castro Gallo. [Grabación de audio]. UAA, 06 de enero de 2016.

Cervantes, T. (2016). Entrevista personal "Aprendizajes de participar en los procesos de evaluación externa" con la licenciada Tania Melina Cervantes Rincón. [Grabación de audio]. UAA, 07 de enero de 2016.

López, F. (2016). Entrevista personal "Aprendizajes de participar en los procesos de evaluación externa" con el maestro Francisco Javier López López. [Grabación de audio]. UAA, 07 de enero de 2016.

López, J. (2016). Entrevista personal "Aprendizajes de participar en los procesos de evaluación externa" con el doctor Jorge Luis López Rodríguez. [Grabación de audio]. UAA, 07 de enero de 2016.

Marín, J. (2016). Entrevista personal “Aprendizajes de participar en los procesos de evaluación externa" con el maestro José Trinidad Marín Aguilar. [Grabación de audio]. UAA, 07 de enero de 2016.

Mosqueda, R. (2016). Entrevista personal “Aprendizajes de participar en los procesos de evaluación externa" con el maestro Ricardo Mosqueda Villalobos. [Grabación de audio]. UAA, 06 de enero de 2016. 